\title{
Role of the TLR4 pathway in blood-spinal cord barrier dysfunction during the bimodal stage after ischemia/reperfusion injury in rats
}

Xiao-Qian Li, Huang-Wei Lv, Wen-Fei Tan, Bo Fang, He Wang and Hong Ma*

\begin{abstract}
Background: Spinal cord ischemia-reperfusion (I/R) involves two-phase injury, including an initial acute ischemic insult and subsequent inflammatory reperfusion injury, resulting in blood-spinal cord barrier (BSCB) dysfunction involving the TLR 4 pathway. However, the correlation between $T L R_{4} / M_{1} D_{88}$-dependent and TLR $/ T R I F-d e p e n d e n t$ pathways in BSCB dysfunction is not fully understood. The aim of this study is to characterize inflammatory responses in spinal cord I/R and the events that define its clinical progression with delayed neurological deficits, supporting a bimodal mechanism of injury.
\end{abstract}

Methods: Rats were intrathecally pretreated with TAK-242, MyD 88 inhibitory peptide, or Resveratrol at a $12 \mathrm{~h}$ interval for 3 days before undergoing 14-minute occlusion of aortic arch. Evan's Blue (EB) extravasation and water content were detected at $6,12,18,24,36,48$, and $72 \mathrm{~h}$ after reperfusion. EB extravasation, water content, and NF-kB activation were increased with time after reperfusion, suggesting a bimodal distribution, as maximal increasing were detected at both 12 and $48 \mathrm{~h}$ after reperfusion. The changes were directly proportional to $T L R_{4}$ levels determined by Western blot. Double-labeled immunohistochemical analysis was also used to detect the relationship between different cell types of BSCB with TLR . Furthermore, NF-KB and IL-1 $\beta$ were analyzed at 12 and $48 \mathrm{~h}$ to identify the correlation between $M y D_{88}$-dependent and TRIF-dependent pathways.

Results: Rats without functional TLR and $\mathrm{MyD}_{88}$ attenuated BSCB leakage and inflammatory responses at $12 \mathrm{~h}$, suggesting the ischemic event was largely mediated by $\mathrm{MyD}_{88}$-dependent pathway. Similar protective effects observed in rats with depleted $\mathrm{TLR}_{4}, \mathrm{MyD}_{88}$, and TRIF receptor at $48 \mathrm{~h}$ infer that the ongoing inflammation which occurred in late phase was mainly initiated by TRIF-dependent pathway and such inflammatory response could be further amplified by $\mathrm{MyD}_{88}$-dependent pathway. Additionally, microglia appeared to play a major role in early phase of inflammation after I/R injury, while in late responding phase both microglia and astrocytes were necessary.

Conclusions: These findings indicate the relevance of $T L R_{4} / M_{y} D_{88}$-dependent and TLR $/ T R I F$-dependent pathways in bimodal phases of inflammatory responses after I/R injury, corresponding with the clinical progression of injury and delayed onset of symptoms. The clinical usage of $\mathrm{TLR}_{4}$ signaling inhibitors at different phases may be a therapeutic option for the prevention of delayed injury.

Keywords: Blood spinal cord barrier, Myeloid differentiation factor 88, Spinal cord ischemia-reperfusion injury, TIR domain-containing adaptor-inducing IFN- $\beta$, Toll-like receptors 4

\footnotetext{
* Correspondence: mahong5466@yahoo.com

Department of Anesthesiology, First Affiliated Hospital, China Medical

University, Shenyang 110001, Liaoning, China
} 


\section{Introduction}

Spinal cord ischemic/reperfusion (I/R) injury is a serious complication of thoracoabdominal aortic surgery. The injury occurs as a two-phase process that correlates with its onset and delayed clinical progression. The first phase includes metabolic and inflammatory processes following ischemia, and the second one is a set of amplified inflammatory responses mediated by biochemical cytokines after restoration of spinal cord blood flow [1,2]. Clinically, this is observed as the bimodal distribution of paraplegia, in which lower limb movement is observed immediately after operation but later deteriorates [3-5]. This phenomenon was reported to occur in $2 \%$ to $18 \%$ of patients, and a total of $50 \%$ of patients displayed delayed neurological deficits due to multifactorial causes [5]. The blood-spinal cord barrier (BSCB), consisting of continuous capillary endothelial cells surrounded by astrocytes and pericytes as well as perivascular microglia, is critical to maintain spinal cord homeostasis and neurologic function [6,7]. Impairment of the BSCB may induce spinal cord edema and progressive breakdown of $\mathrm{BSCB}$ integrity, which may aggravate injury, resulting in paraplegia or even death, thus highlighting the complexity of the injury $[1,3,8,9]$. Previous studies have demonstrated that Toll-like receptors $4\left(\mathrm{TLR}_{4}\right)$, a class of transmembrane proteins that can recognize specific ligands extracellularly, are closely associated with the inflammation that occurs after I/R injury and mediate the pathogenesis of hind-limb function and neuronal viability [10-12]. The myeloid differentiation factor $88\left(\mathrm{MyD}_{88}\right)$ and TIR domain-containing adaptorinducing IFN- $\beta$ (TRIF), which function via the up regulation of $\mathrm{TLR}_{4}$, are the two most important adaptor proteins that provoke the transduction of common downstream signaling molecules, such as NF- $\mathrm{kB}$, into the nucleus and regulate the expression of target inflammatory genes [13-15]. In the $\mathrm{MyD}_{88}$-dependent pathway, previous studies have demonstrated that $\mathrm{TLR}_{4}$ directly activates $\mathrm{MyD}_{88}$ and thus facilitates further activation of NF-kB and downstream inflammatory cytokine interleukin (IL)- $1 \beta$ in the early phase $[10,13,16]$. However, recent studies suggest that $\mathrm{TLR}_{3}$ is not indispensable for the TRIF-dependent pathway [17]. In the $\mathrm{TLR}_{4}$ signaling pathway, TRIF is recruited upon receiving stimuli from TICAM-2, leading to rapid activation of interferon regulatory factor 3 and beta interferon, which in turn activates TRIF and $\mathrm{MyD}_{88}$ [15-17] and induces delayed NF-kB activation [18]. In our previous studies, we have reported the breakdown of BSCB integrity in the spinal cord after $I / R$ injury $[8,9]$. Therefore, procedures that can preserve the intact function of the BSCB and attenuate the inflammatory responses after I/R injury would improve prognosis. However, not much is known about the underlying roles of $\mathrm{TLR}_{4}$ signaling and the transduction of its downstream adaptor receptors. The aim of this study is to investigate the role of $\mathrm{TLR}_{4}$ in increased BSCB leakage using a specific cell population during a post-injury bimodal stage. We established a functional deletion of $\mathrm{TLR}_{4}, \mathrm{MyD}_{88}$, and TRIF receptor by intrathecal treatment with TAK-242 [19], $\mathrm{MyD}_{88}$ inhibitory peptide (MIP) [16], or Resveratrol [20] to prevent TLR 4 from interacting with its downstream receptors, namely, $\mathrm{MyD}_{88}$ and TRIF. In addition, we explored the underlying relevance of $\mathrm{TLR}_{4} / \mathrm{MyD}_{88}$-dependent and $\mathrm{TLR}_{4} / \mathrm{TRIF}$ dependent pathways in the bimodal phase of inflammatory responses after $I / R$ injury by determining the activation of $\mathrm{NF}-\mathrm{kB}$ and subsequent products of the proinflammatory cytokine, IL-1 $\beta$.

\section{Materials and methods}

\section{Experimental animals}

All experimental procedures were approved by the Ethics Committee of China Medical University and were performed in accordance with the Guide for the Care and Use of Laboratory Animals (U.S. National Institutes of Health publication No. 85-23, National Academy Press, Washington DC, revised 1996). The rats used in this study were all male Sprague-Dawley rats weighing between 200 and $250 \mathrm{~g}$, neurologically intact before anesthesia, and expressed normal, functional TLR 4 . The rats were bred in standard cages with free access to food and water, and were housed separately after surgery at the First Affiliated Hospital, China Medical University.

\section{Animal surgical procedures}

The spinal cord $\mathrm{I} / \mathrm{R}$ model was induced by occluding the aortic arch for $14 \mathrm{~min}$, as previously reported [21]. In general, all rats were anesthetized with an intraperitoneal injection of $4 \%$ sodium pentobarbital at an initial dose of $50 \mathrm{mg} / \mathrm{kg}$. The aortic arch was exposed through a cervicothoracic approach. Under direct visualization, the aortic arch was cross-clamped between the left common carotid artery and the left subclavian artery. A laser Doppler blood flow monitor (Moor Instruments, Axminster, Devon, UK) was used to confirm complete occlusion. Ischemia was confirmed as a $90 \%$ decrease in flow measured at the femoral artery that continued for $14 \mathrm{~min}$, after which the clamps were removed and followed by $72 \mathrm{~h}$ of reperfusion. Sham operation rats underwent the same procedure, but no occlusion of the aortic arch was performed.

\section{Experimental protocol}

Rats were randomly assigned to one of the five groups: Sham, Ischemia/reperfusion (I/R), MIP, Resveratrol, and TAK-242 (TAK) group. Continuous intrathecal injections were performed at $12 \mathrm{~h}$ intervals for 3 days prior to ischemia or the induction of sham surgery. In order to study the role of $\mathrm{TLR}_{4}$-mediated signal pathways in $\mathrm{I} / \mathrm{R}$ injury, the rats were intrathecally injected with $10 \mu \mathrm{L}$ 
MIP $(50 \mathrm{nmol} / \mu \mathrm{L})$, Resveratrol $(50 \mathrm{nmol} / \mu \mathrm{L})$, TAK-242 $(10 \mathrm{nmol} / \mu \mathrm{L})$, or an equivalent volume of normal saline as control at $\mathrm{L}_{4-6}$ segments of the spinal cord, as previously described $[16,19,20]$.

\section{Examination of blood-spinal cord barrier leakage}

Measuring the water content of the spinal cord and Evan's Blue (EB) extravasation were, respectively, the most common methods used for the quantitative and qualitative examination of BSCB leakage after spinal cord $I / R$ injury. The percentage of water content was calculated as: (wet weight - dry weight)/wet weight $\times 100$, using a wet-dry method.

For quantification of EB extravasation, $30 \mathrm{~g} / \mathrm{L} E B$ (45 mg/kg; Sigma, St. Louis, MO, USA) was slowly administered intravenously in the tail vein 60 minutes before sacrificing the animals. After soaking the tissues in methanamide for $24 \mathrm{~h}\left(60^{\circ} \mathrm{C}\right)$ and centrifugation at $20,000 \times g$ for $20 \mathrm{~min}$, the absorption of the supernatant was detected at $632 \mathrm{~nm}$ and reported as the amount of EB per wet tissue weight $(\mu \mathrm{g} / \mathrm{g})$. For dye fluorescence measurements, the tissue was fixed in $4 \%$ paraformaldehyde, then sectioned $(10 \mu \mathrm{m})$ and visualized using a BX-60 (Olympus, Melville, NY, USA) fluorescence microscope (green zone).

\section{Immunofluorescence staining}

Double immunofluorescence analysis was performed to measure the activation and localization of $\mathrm{TLR}_{4}$ after I/R injury. The spinal cord was fixed and sectioned into $10-\mu \mathrm{m}$ slices with a Leica CM3050 S cryostat. The sections were blocked with $10 \%$ bovine serum albumin for $1 \mathrm{~h}$ at room temperature and incubated overnight at $4^{\circ} \mathrm{C}$ with the primary antibodies: mouse anti-TLR 4 (1:100, Abcam, Cambridge, UK), rabbit anti-CD31 antibody (1:800, Abcam), rabbit anti-CD13 antibody (1:500, Abcam), rabbit antiIba-1 antibody (1:800, Wako, Germany), and rabbit antiGFAP antibody (1:800, Abcam). After incubation with Alexa 594-conjugated donkey anti-mouse IgG (1:500, Molecular Probes, Eugene, Oregon, USA) and Alexa 488-conjugated donkey anti-rabbit IgG (1:500, Molecular Probes) for $2 \mathrm{~h}$ at room temperature, the images were captured using a Leica TCS SP2 (Leica Microsystems, Buffalo Grove, IL, USA) laser scanning microscope and photographed by the attached digital camera to determine the number of immunoreactive cells. Non-specific staining was determined by omitting the primary antibody. The data were expressed as numbers of positive cells/area/ spinal section \pm standard error mean (SEM).

\section{Biochemical analysis}

The spinal cord was collected, homogenized, and centrifuged. The level of IL-1 $\beta$ was determined by ELISA kit (R \& D Systems, Minneapolis, MN, USA). According to the manufacturer's instructions, the absorbance was detected at $450 \mathrm{~nm}$ and the content of each sample was calculated from the standard curve and the quantity of IL- $1 \beta$ was expressed as $\mathrm{pg} / \mathrm{mg}$ protein.

\section{Western blot analysis}

The expression of $\mathrm{TLR}_{4}, \mathrm{MyD}_{88}$, TRIF, and NF-kB p65 in spinal cord tissue were determined by Western blot. The rats' spinal cords were homogenized, and the total proteins were purified using cell and tissue protein extraction reagents according to manufacturer's instructions (KC415; KangChen, Shanghai, China). The antibodies used in this experiment were mouse monoclonal anti-TLR 4 (1:500, Abcam), rabbit polyclonal anti-MyD $\mathrm{D}_{88}$ (1:500, Abcam), rabbit polyclonal anti-TRIF (1:500, Abcam), and rabbit polyclonal anti- NF-kB p65 (1:500, Abcam), along with horseradish peroxidase conjugated secondary antibodies (Bioss, Beijing, China). Semi-quantitation of scanned images was performed using Quantity One software (Bio-Rad Laboratories, Milan, Italy).

\section{Statistical analysis}

All data were expressed as means \pm standard error mean (means \pm SEM) and analyzed by SPSS software (version 17.0, SPSS Inc., Chicago, IL, USA). All variables measured in this study were normally distributed, and the groups were compared with Student's $t$-test or one-way analysis of variance (ANOVA), followed by NewmanKeuls post-hoc analysis. A $P$ value of $<0.05$ was considered statistically significant.

\section{Results}

\section{Effects of BSCB leakage after I/R}

To determine the time frame of BSCB leakage and explore the underlying mechanisms, we performed a time course of I/R injury from 6 to $72 \mathrm{~h}$ post-operation determined by the extravasation of EB dye, which was visualized as red fluorescence under the fluorescent microscope (Figure 1A). Since the $\mathrm{L}_{4-6}$ segments of the spinal cord are most vulnerable to ischemic injury [22], the increased BSCB leakage was analyzed at this site. Our observations suggest that the maximal differences in EB extravasations between the Sham group and I/R group occurred at 12 and $48 \mathrm{~h}$ after surgery. Quantification of EB content in the injured spinal cord revealed that it reached maximal EB extravasations in the I/R group at $12 \mathrm{~h}$, followed by delayed and aggravated BSCB leakage beginning at $36 \mathrm{~h}$, peaking again at $48 \mathrm{~h}$ (Figure 1B). Similarly, differences in water content attributed to the increased BSCB leakage in the I/R group also had a bimodal distribution, peaking at 12 and $48 \mathrm{~h}$ (Figure $1 \mathrm{C}$ ). There were significant differences between the two groups at all observed time points $(P<0.05$ vs. Sham group). 


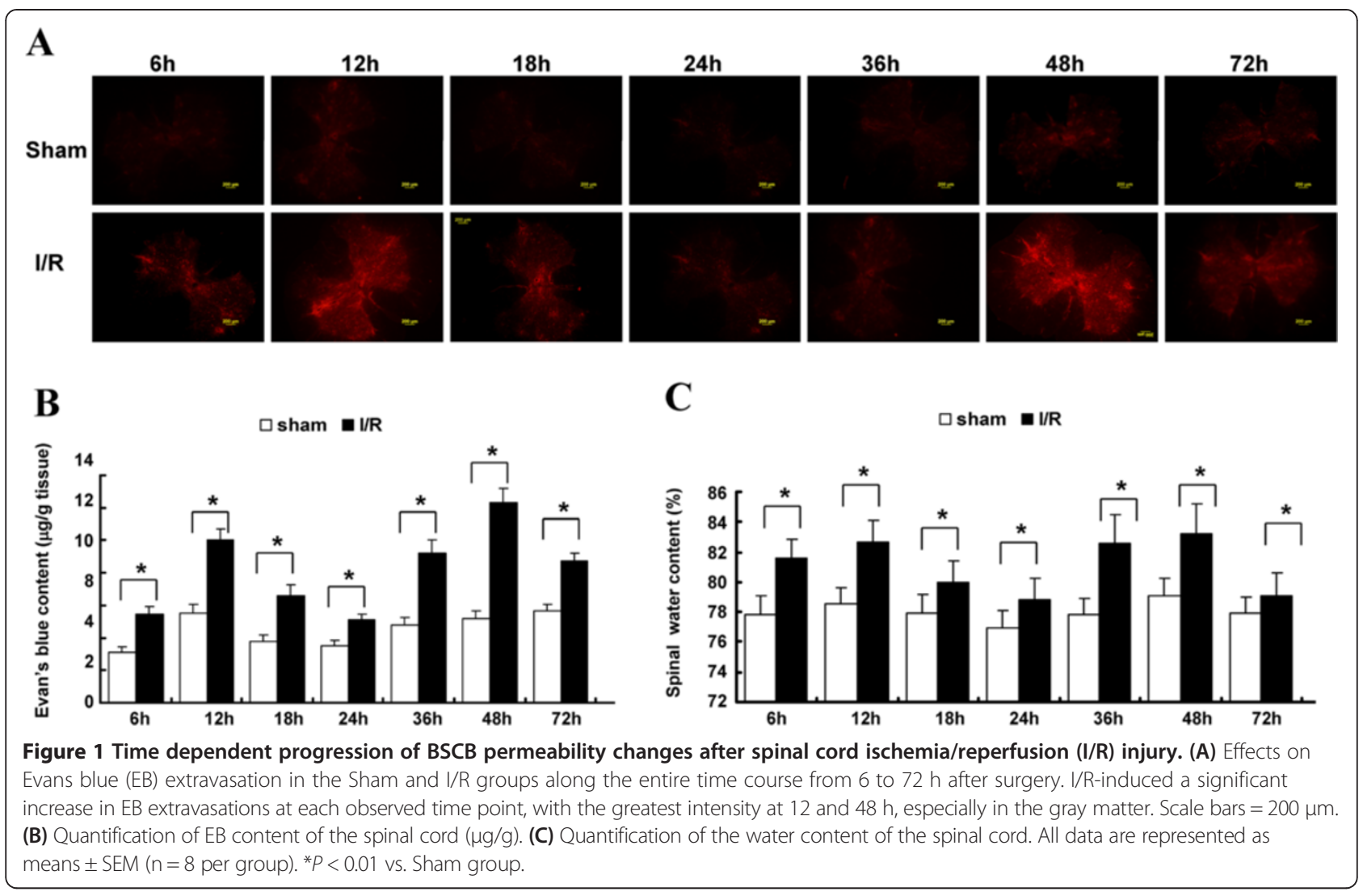

\section{Effects of $\mathrm{TLR}_{\mathbf{4}}$ immunoreactivity after I/R}

Immunofluorescence and Western blot analyses for $\mathrm{TLR}_{4}$ performed during the time course of $I / R$ injury were shown in Figure $2 \mathrm{~A}$. The expression of $\mathrm{TLR}_{4}$ significantly increased with time in spinal cords of the I/R group, where the $\mathrm{TLR}_{4}$ protein was expressed at maximum levels at 12 and $48 \mathrm{~h}$ after surgery.

\section{Blocking effects of the $\mathrm{TLR}_{\mathbf{4}}$ pathway after I/R}

We established a functionally compromised $\mathrm{TLR}_{4}, \mathrm{MyD}_{88}$, and TRIF by intrathecal pretreatment with TAK-242, MIP, and Resveratrol, and analyzed the blocking effects of each group at 12 and $48 \mathrm{~h}$ after surgery since BSCB leakage was maximal at these two time points. As the cross-talk in signal transduction used to verify the specification and blocking effects of the inhibitors, the changes in protein level of TLR $4, \mathrm{MyD}_{88}$, and TRIF were detected by Western blot (Figure 3A-C). Quantitative Analysis showed that I/R injury induced $\mathrm{TLR}_{4}, \mathrm{MyD}_{88}$, and TRIF expressions in all groups compared to the Sham group at 12 and $48 \mathrm{~h}$ after injury (Figure $3 \mathrm{D}-\mathrm{F}$, all $P<0.05$ vs. Sham group). Pretreatment with TAK-242 effectively prevented the up regulation of $\mathrm{TLR}_{4}$ without much influence on expressions of $\mathrm{MyD}_{88}$ and TRIF, suggesting satisfactory specificity and effectiveness of TAK-242 (Figure 3A and D, $P<0.05$ ). Likewise, following targeted down regulation of $\mathrm{MyD}_{88}$ and
TRIF by intrathecal pretreatment with MIP and Resveratrol, respectively, there were no significant differences in expressions of the other two indicators TLR $_{4}$ and TRIF or $\mathrm{TLR}_{4}$ and $\mathrm{MyD}_{88}$ ) (Figure $3 \mathrm{~B}$ and $\mathrm{E}$ vs $\mathrm{C}$ and $\mathrm{F}$, both $P>0.05)$.

Effects of the $\mathrm{TLR}_{4}$ pathway on colocalization of different cell types in blood spinal cord barrier after I/R

Continuous capillary endothelial cells, pericytes, astrocytes, and perivascular microglia are the major cellular components of the BSCB. With the understanding that the $\mathrm{TLR}_{4}$ pathway may play an important role in the inflammatory damage to BSCB integrity, we further identified a specific cell population in BSCB with the following cellular markers: CD31 (platelet endothelial cell adhesion molecule-1, capillary endothelial cell marker), CD13 (aminopeptidase-N, pericyte marker), Iba-1 (microglial marker), and GFAP (astrocyte marker) (Figure 4). Based on our results, the majority of $\mathrm{TLR}_{4}$ was co localized with the distribution of Iba-1-positive microglia in rats of the $\mathrm{I} / \mathrm{R}$ group at both 12 and $48 \mathrm{~h}$ after surgery, but not in sham-operated ones, confirming that $\mathrm{TLR}_{4}$ was indeed upregulated in microglia. Similarly, the increasing numbers of double-labeled astrocytes were found in rats of the I/R group at $48 \mathrm{~h}$ after surgery, suggesting the involvement of astrocytes. However, no identical fluorescence label 


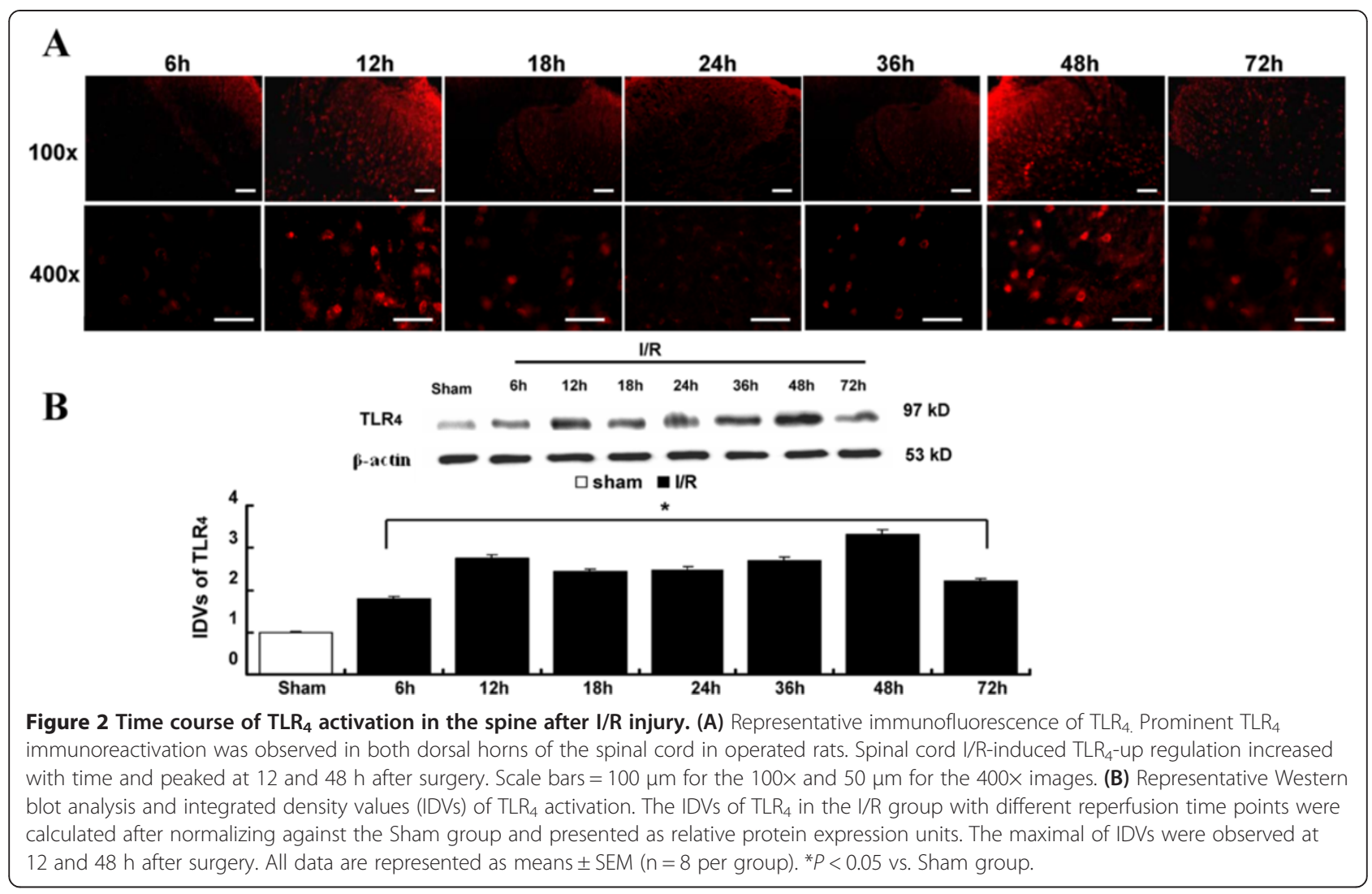

of $\mathrm{TLR}_{4}$ was found in capillary endothelial cells and pericytes of rats undergoing ischemia or sham-operation at the above time points. Quantification of $\mathrm{TLR}_{4}$ colocalization in Figure $4 \mathrm{C}$ confirmed that the majority of $\mathrm{TLR}_{4}$ was expressed in microglia at $12 \mathrm{~h}$ after injury, while at $48 \mathrm{~h}$ after surgery, $\mathrm{TLR}_{4}$ was comparably expressed in both microglia and astrocytes (all $P<0.01$ vs. Sham group).
Additionally, we determined the effects of the $\mathrm{TLR}_{4}$ pathway on double-labeled microglia and astrocytes at $48 \mathrm{~h}$ after I/R injury for the most double-labeled cells observed at that time. As shown in Figure 4B, the maximal number of double-labeled microglia and astrocytes were both observed in the I/R group. Compared with $\mathrm{I} / \mathrm{R}$ group, the number of double-labeled microglia and astrocytes were decreased the most in the TAK group and

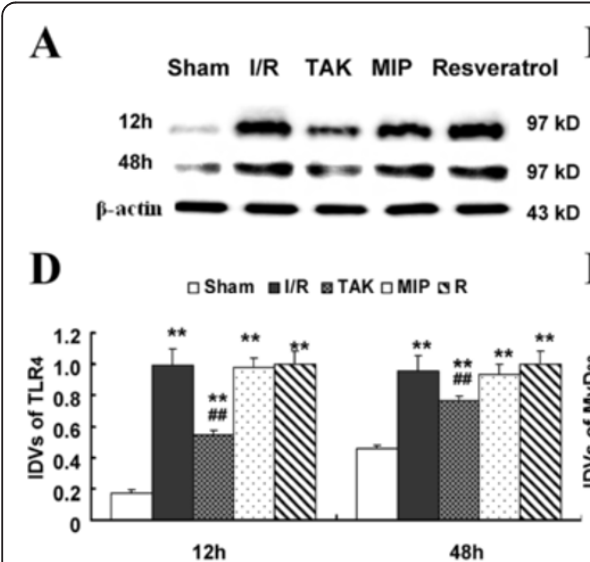

\section{B}

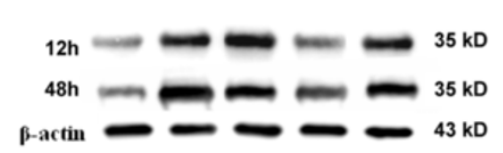

$\mathbf{E}$

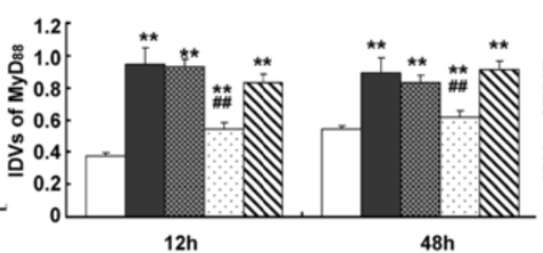

C

\section{Sham I/R TAK MIP Resveratrol}

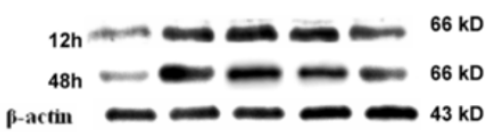

F

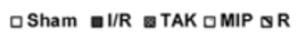

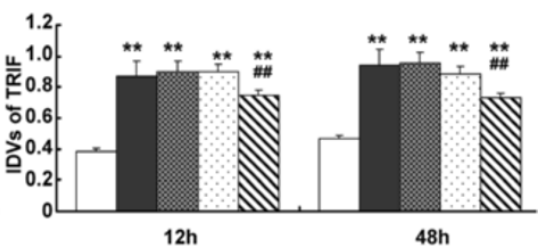

Figure 3 Blocking effects of specific binding protein on $\mathrm{TLR}_{\mathbf{4}}, \mathrm{MyD}_{\mathbf{8 8}}$, and TRIF protein. (A-C) Representative Western blot analysis of TLR $\mathrm{MyD}_{88}$, and TRIF protein in $\mathrm{L}_{4-6}$ segments of spinal cord at 12 and $48 \mathrm{~h}$ after $\mathrm{I} / \mathrm{R}$ injury. (D-F) The integrated density values (IDVs) in each group with different reperfusion time points were calculated after normalizing against $\beta$-actin and presented as relative protein expression units. Data are presented as means \pm SEM ( $n=8$ per group). ${ }^{* *} P<0.01$ vs. Sham group; \#\# $P<0.01$ vs. I/R group. 


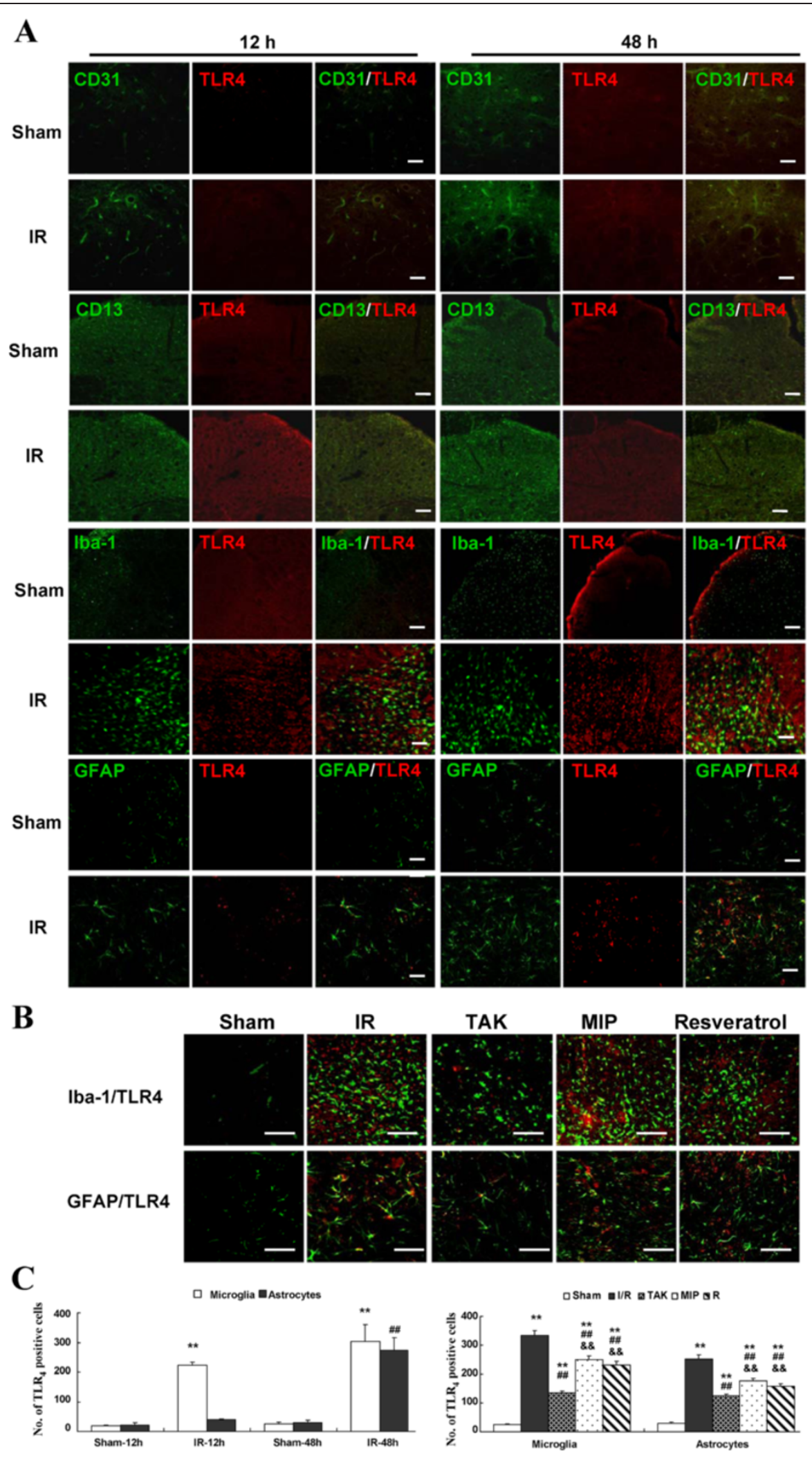

Figure 4 (See legend on next page.) 
(See figure on previous page.)

Figure 4 Double-labeled immunofluorescence $\mathrm{TLR}_{4}$ receptor and specific cell population in blood spinal cord barrier after spinal cord I/R injury. (A) Representative immunohistochemical localization of capillary endothelial cells (CD31; green), pericytes (CD13; green), microglia (Iba-1; green), astrocytes (GFAP; green), and Toll-like receptor (TLR 4 ; red) in spinal cords of sham-operated or operated rats at 12 and $48 \mathrm{~h}$ after the surgical procedure, respectively. Scale bars $=100 \mu \mathrm{m}$ for the 200x images. (B) Effects of TLR 4 signaling on TLR4 co-localization cells Representative double-labeling micrographs show that intrathecal pretreatment with TAK-242, MIP, and Resveratrol before I/R injury significantly prevented microglia and astrocytes upregulated expression of $\mathrm{TLR}_{4}$ at $48 \mathrm{~h}$ after the surgery. Scale bars $=50 \mu \mathrm{m}$ for the $400 \times$ images. (C) Quantification of TLR ${ }_{4}$-positive microglia and TLR4-positive astrocytes in the spinal cords. Data are presented as mean $\pm S E M(n=6) .{ }^{*} P<0.01$ vs. Sham group; \#\# $P<0.01$ vs. I/R group; \&\& $P<0.05$ vs. TAK group.

the least in the MIP group $(P<0.05$ vs. I/R group, $P>0.05$ vs. MIP group). Almost no double-labeled glial cells were detected in the Sham group at the above time point.

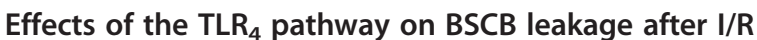

We next determined the role of the $\mathrm{TLR}_{4}$ signaling cascade in BSCB leakage. As shown in Figure 5A, maximal leakage, as monitored by amount of EB extravasation, was noted in the I/R group at 12 and $48 \mathrm{~h}$ after surgery $(P<0.01$ vs. Sham group). Intrathecal pretreatment with TAK-242 prevented the increased leakage of BSCB at both time points recorded (both $P<0.05$ vs. I/R group). Comparing EB extravasation among groups, highly intense red fluorescence was observed in the $I / R$ and Resveratrol groups at $12 \mathrm{~h}$, which intensified at $48 \mathrm{~h}$ in all groups, especially in the gray matter of the $I / R$ and MIP groups. Minimal EB extravasation was detected in the Sham group at the above two time points $(P<0.05$ vs. I/R group).

Additionally, assessments of water content showed similar results at 12 and $48 \mathrm{~h}$ after surgery, as seen in Figure $4 C$. It could be inferred that $I / R$ increased the water content due to spinal cord edema $(P<0.05$ vs. Sham group), while intrathecal transplantation with TAK242 attenuated the increase in water content $(P<0.05$ vs. $\mathrm{I} / \mathrm{R}$ group). Of note, the depletion of $\mathrm{MyD}_{88}$ by intrathecal transplantation with MIP only attenuated these effects at $12 \mathrm{~h}$ ( $P<0.05$ vs. I/R group); a similar effect was observed at $48 \mathrm{~h}$ in the group lacking TRIF function $(P<0.05 \mathrm{vs}$. I/R group).

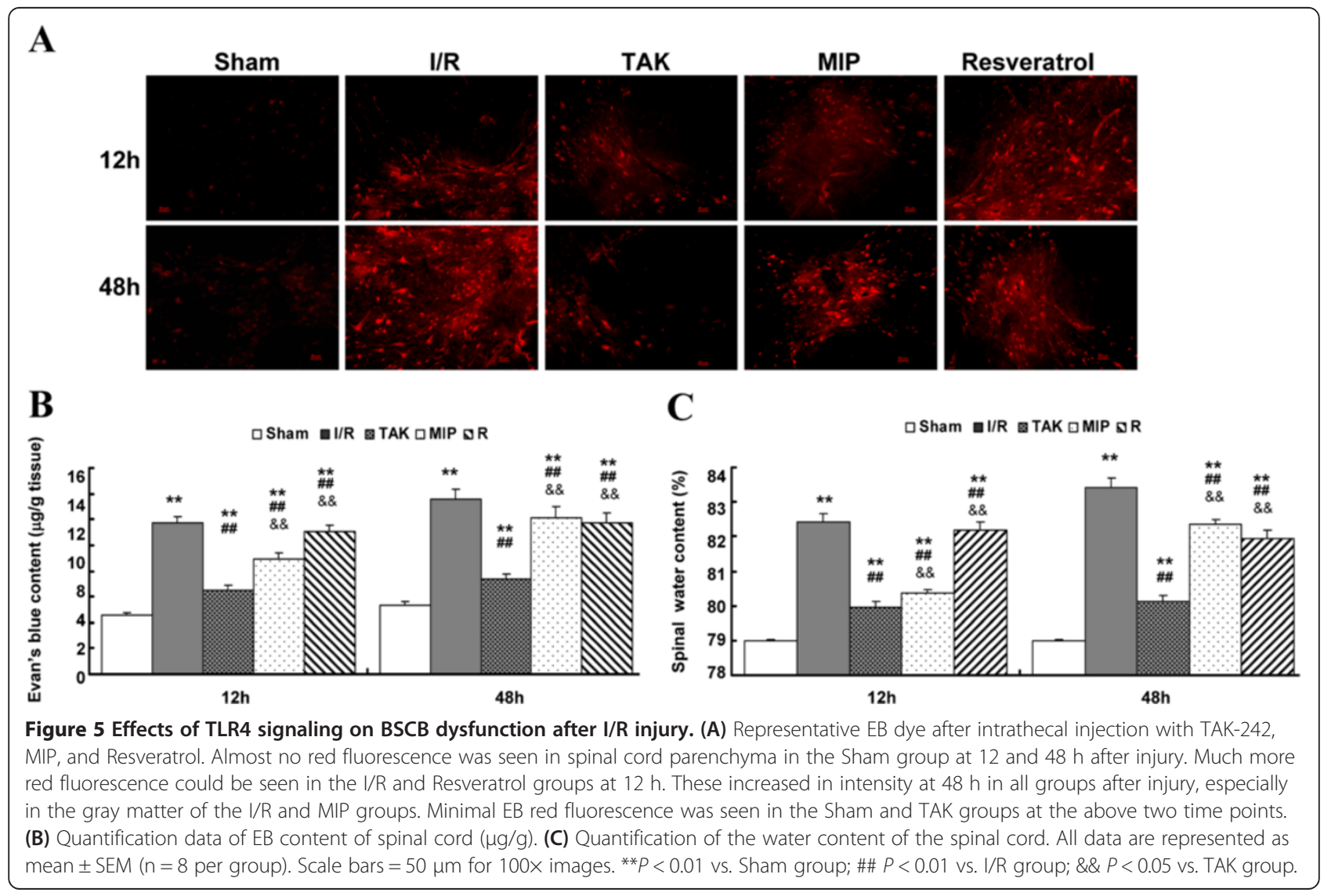




\section{Effects of the $\mathrm{TLR}_{4}$ pathway on NF-KB activation and inflammatory cytokines after I/R}

Finally, we examined the levels of NF- $\mathrm{kB}$ and its downstream inflammatory cytokine proteins after intrathecal pretreatment with TAK-242, MIP, or Resveratrol. As presented in Figure 6, I/R injury greatly induced NF- $\mathrm{B}$ in comparison with the sham group at 12 and $48 \mathrm{~h}$ after surgery $(P<0.05$ vs. Sham group), and such induction was accompanied with significantly increased IL-1 $\beta$ $(P<0.05$ vs. Sham group). Pretreatment with TAK-242 inhibited NF- $\mathrm{kB}$ and IL-1 $\beta$ expression in the spinal cord after injury at both time points $(P<0.05$ vs. I/R group). To compare the effects on NF- $\mathrm{KB}$ and IL- $1 \beta$ after pretreatment with MIP or Resveratrol, NF- $\mathrm{kB}$ and IL-1 $\beta$ were detected in the $I / R$ group and the Resveratrol group at $12 \mathrm{~h}$; both NF-kB and IL- $1 \beta$ were significantly increased in all groups at $48 \mathrm{~h}$, especially in the rats of the I/R and MIP groups $(P<0.05$ vs. I/R group).

\section{Discussion}

Spinal cord I/R injury after thoracic aortic surgery is invariably associated with dysfunction of the BSCB and plays a fundamental role in the progression of several

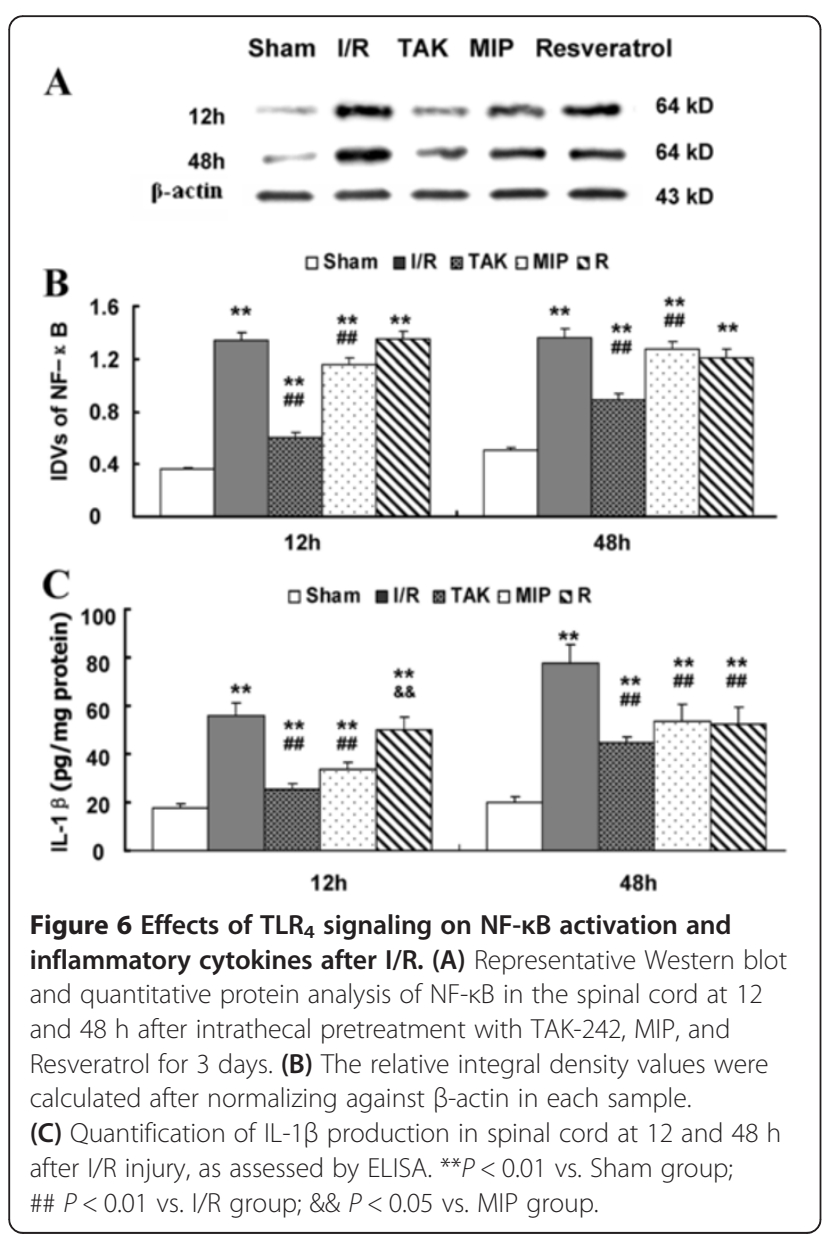

unpredictable and disastrous complications, such as spinal cord swelling and secondary nerve injury, which account for much of the morbidity and mortality of this condition $[4,5]$. Spinal cord edema is often long lasting and resistant to therapeutic intervention. Recently, TLRs, especially $\mathrm{TLR}_{4}$, have gained extensive attention for their important roles in various models of $I / R$ injury $[1,18]$. In our previous study, we demonstrated that increased activation of $\mathrm{TLR}_{4}$ in the spinal cord was associated with increases of BSCB leakage after I/R injury. Our present study is the first attempt to further characterize the inflammation that occurs in a specific cell population after spinal cord $I / R$ injury and suggests the functional relevance of $\mathrm{TLR}_{4}$-mediated downstream signal transduction during the bimodal stage after injury.

With an improved understanding of spinal cord I/R injury, the damage sustained during thoracoabdominal aortic occlusion has been classically defined as an initial ischemic event and a delayed reperfusion injury, corresponding with the clinical presentation of bimodal distribution of spinal cord injury [1-3]. Clinically, the manifestation of immediate severe spinal cord injury is rare. Usually, patients experience delayed neurological deficits and develop paraplegia in the several hours to days after surgery, which highlights the pathological mechanisms defined by the responses in the ischemic phase, and an ongoing amplification of molecular insults during the reperfusion phase [23]. After I/R injury, the cytokines released by local cellular populations in the early phase may cause increased accumulation of inflammatory cells, and the chemokines released amplify inflammatory responses, leading to the clinical presentation of functional deficits $[24,25]$. As demonstrated in our previous study, increased BSCB leakage closely associated with invasion of exogenous ligands inducing immune and inflammatory responses were found during the course of spinal cord I/R injury, as evaluated by extravasation of EB dye $[8,9]$. Similarly, in this study, the bimodal distribution of EB extravasations was clearly observed as two phases, one stage from 6 to $18 \mathrm{~h}$ after surgery which continued for several hours, and the later one began at $36 \mathrm{~h}$ and peaked at $48 \mathrm{~h}$ (Figure 1), paralleling the clinical manifestation of an early ischemic phase with great risks of delayed and exacerbated injury, such as paralysis, two days after surgery $[4,5]$.

A growing body of evidence indicates the important role of $\mathrm{TLR}_{4}$ in the evolution of an inflammatory reaction after $I / R$ injury $[16,19]$. The stimulation from ischemic or necrotic cells causes $\mathrm{TLR}_{4}$ activation, subsequent translocation of NF- $\mathrm{KB}$ to the nucleus, and the production of proinflammatory cytokines and chemokines [10,16]. Similarly, the immunohistochemistry data in our study confirm the essential role of $\mathrm{TLR}_{4}$ in the inflammatory processes associated with increased BSCB leakage. Significantly, our study lies in the understanding 
of the correlation between the $\mathrm{TLR}_{4}$-mediated $\mathrm{MyD}_{88^{-}}$ dependent and TRIF-dependent signaling pathways during the bimodal stage after I/R injury. Therefore, we chose 12 and $48 \mathrm{~h}$ time points at which maximal changes were reached after $I / R$ injury to further explore the role of $\mathrm{TLR}_{4}$-mediated downstream signaling. It is essential to develop potential therapies targeting these points to attenuate and avoid subsequent injury. Some investigations have already reported the effects of TAK-242, MIP, and Resveratrol on the inhibiting functions of TLR and its downstream adaptor receptors, $\mathrm{MyD}_{88}$ and TRIF, which receive the stress stimulation $[16,19,20]$. In this study, we depleted the function of the $\mathrm{TLR}_{4}$ signaling pathway by continuous intrathecal injection with TAK242, MIP, or Resveratrol at a 12-h interval 3 days before surgery. The effects of the inhibitors were detected by Western blot. Based on our results, the protein levels (Figure $3 \mathrm{~A}-\mathrm{C}$ ) and integrated density values (Figure 3D-F) of $\mathrm{TLR}_{4}, \mathrm{MyD}_{88}$, and TRIF in the I/R group were obviously higher than those in rats intrathecally pretreated with TAK-242, MIP, or Resveratrol, as well as those in the Sham group at both observed time points. Therefore, TAK-242, MIP, and Resveratrol employed before reperfusion significantly prevented their functions from receiving the reperfusion-induced stimulation. However, pretreatment with only one of the inhibitors lead to changes in three indicators ( $\mathrm{TLR}_{4}, \mathrm{MyD}_{88}$, and TRIF) in each group simultaneously. Therefore, it is important to further assure specificity of each inhibitor by detecting whether there is cross-talk in signal transduction. As tested herein, TAK242 specifically binds the $\mathrm{TLR}_{4}$ receptor without much impact on the functions and expressions of adaptor receptors $\mathrm{MyD}_{88}$ and TRIF downstream. Likewise, MIP and Resveratrol were proven to solely decrease the protein expression of $\mathrm{MyD}_{88}$ and TRIF, respectively, demonstrating the reliability of intrathecal injection with specific binding proteins to explore the role of the $\mathrm{TLR}_{4}$ pathway, as performed in our study (Figure 3).

Our study showed that $I / R$ injury caused vascular events of BSCB in segments most vulnerable to ischemia and exhibited more pronounced vascular disruptions at $48 \mathrm{~h}$ than those at $12 \mathrm{~h}$ after surgery. The BSCB act as a metabolic barrier to strictly regulate molecular exchange between the circulating blood and spinal cord, and strictly controls the spinal cord microenvironment required for normal neuronal function and micro environmental homeostasis $[6,9,26]$. Increased BSCB leakage would expose the spinal cord to endogenous ligands and exogenous invading pathogens in the circulation and aggravate inflammatory responses and neurotoxic effects in the development and recovery associated with spinal cord injury, resulting in irreversible damage to the physiological functions of aesthesia and kinesis, as well as to bladder, bowel, and sexual functions following the injured plane. Moreover, the BSCB may determine the innate immune reaction [26]. Therefore, the exploration of the cellular compositions of the BSCB implicated in $\mathrm{TLR}_{4}$ activation will greatly contribute to the better understanding the physiological function or pathological development in the early and late phases of inflammation after I/R injury. As reported in previous studies, glial activation occurred in response to ischemia and the responses were very rapid, especially in microglia, which changed in morphology and increased in number within the first few minutes to hours after reperfusion $[1,3,7,26]$, while astrocytes were observed to be mildly increased over $8 \mathrm{~h}$ after reperfusion [26]. Similarly, the microglia increased in number and showed intense staining in the spinal cord after surgery and peaked at 12 and $48 \mathrm{~h}$, whereas the GFAP staining became intense $24 \mathrm{~h}$ after reperfusion and peaked at $48 \mathrm{~h}$, suggesting that increased BSCB leakage may be attributed to glial activation through their membrane-bound receptor $\mathrm{TLR}_{4}$ which is closely associated with inflammatory reactions (Figure 4). Increasing evidence has shown that there is an important and complicated signal transmission within activated neuralgia in response to spinal cord injury $[6,7,18]$. To explain the different activation phases of glial cells, one can easily consider the different degrees of BSCB leakage and the different roles of glial cells involved. Perivascular microglia surrounding the BSCB are considered as the first actors to remove tissue, cells debris, or macromolecular proteins penetrating through functional leakage of the BSCB, as suggested in the model of spinal cord ischemia and compressive injury [7,26,27]. Astrocytes have been demonstrated to play a major role in homeostasis of the BSCB and recovery of neurological function after ischemia [7]. In the present study, compared with the widespread activation of microglia both in the early (12 h) and late phase $(48 \mathrm{~h})$ after surgery, the increases in GFAP immunoreactivity were mainly distributed in spinal gray matter, where the capillaries were more numerous and formed a dense capillary bed in comparison with white matter. Thus, astrocytes were considered to be hyper-responsive states in cases of organic damage of BSCB. In addition, the effects of the inhibitors on double-labeled glial cells were chosen to quantify at $48 \mathrm{~h}$ after surgery for the maximal activation based on micrographs. As shown in Figure $4 \mathrm{~B}$ and $\mathrm{C}$, compared with $\mathrm{I} / \mathrm{R}$ group, the number of double-labeled microglia and astrocytes in groups pretreated with TAK-242, MIP, and Resveratrol, respectively, decreased the most in the TAK group, suggesting that the relatively intact microenvironment of the spinal cord contributed to the weak responses of glial cells in the BSCB lacking the functional $\mathrm{TLR}_{4}$ receptor after surgery. Comparing the effects of MIP and Resveratrol treatment, immunofluorescence staining and quantification data demonstrated that I/R-induced elevated double- 
labeled microglia and astrocytes were significantly decreased in the Resveratrol group, whereas less so in the MIP group, indicating a major involvement of the $\mathrm{TLR}_{4^{-}}$ TRIF-dependent pathway $48 \mathrm{~h}$ after surgery.

Up regulating TLR4 and NF- $\mathrm{KB}$ was accompanied with the production of proinflammatory cytokines. As expected, our results revealed increases in NF-kB and IL- $1 \beta$ with increasing time after $I / R$ injury, signifying the amplified and aggravated inflammatory responses in pathogenesis in the spinal cord. Previous studies have demonstrated the capacity of $\mathrm{TLR}_{3}$ to activate TRIF $[15,28]$ and that $\mathrm{TLR}_{4}$ directly activated $\mathrm{MyD}_{88}[16,19]$. With recent advancements in this area, some studies have indicated that $\mathrm{TLR}_{3}$ is not indispensable for the TRIF-dependent pathway while the TLR 4 receptor has the potential to activate the TRIF-dependent pathway upon receiving a signal from $\mathrm{MyD}_{88}$-dependent pathways [15]. The signals that bridged through TICAM- $_{-2}$ to TICAM-1 led to the recruitment of TRIF and TRAM in a cascade [15,29], resulting in the rapid production of cytokines and the late phase activation of NF- $\mathrm{kB}$ and mitogen-activated protein kinases, thus exerting positive feedback on $\mathrm{MyD}_{88}$-activation [30,31]. Therefore, based on our data, the ongoing cascade of inflammatory responses involves the activation of both the $\mathrm{MyD}_{88}$ and TRIF pathways.

IL-1 $\beta$ is the most commonly implicated protein in inflammatory responses and exhibits biphasic distribution in various models of $I / R$ injury $[2,3,32]$. These studies revealed that IL-1 $\beta$ significantly increased and remained high in the reperfusion period, and that heightened IL-1 $\beta$ were associated with both early ischemic and late reperfusion injury $[2,3,32,33]$. Based on our results, we found analogously biphasic IL- $1 \beta$ responses during the reperfusion period since the levels expressed at 12 and $48 \mathrm{~h}$ were much higher than those at $24 \mathrm{~h}$ and were in accordance with the activation of NF- $\mathrm{kB}$. It was intriguing to explore the temporal relationship between $\mathrm{TLR}_{4} / \mathrm{MyD}_{88}$ and $\mathrm{TLR}_{4} /$ TRIF. Similar to the results in our study, O'Neill and Bowie suggested that the expression of NF- $\mathrm{kB}$ occurred in two phases after $\mathrm{TLR}_{4}$ activation [17]: MyD88 mediated events in the early phase, while TRIF mediated the later phase. We found that there was a significant increase in NF- $\mathrm{KB}$ release and IL- $1 \beta$ production at $12 \mathrm{~h}$. Nevertheless, contrasting results were observed at $48 \mathrm{~h}$ in the Resveratrol group (Figure 4), indicating that $\mathrm{MyD}_{88}$ and TRIF dependence occurred in the early and late phases, respectively.

Of note, sequential activation of microglia and astrocytes were supported by immunofluorescence staining, but not necessarily in that order given the complexity of in vivo experiments. Nevertheless, a sustained majority of $\mathrm{TLR}_{4}$ expression occurs in microglia throughout the inflammatory responses, and the increasing involvement of astrocytes with time in the late phase might be an explanation for the delayed neurological deficits $[3,7,26,27,34]$. Further in vitro studies still need to be conducted to identify the development and mode of action of these inflammatory mediators to better elucidate the mechanism underlying the pathogenesis.

Taken together, our data suggest that there could be different phases of spinal cord I/R injury. Inflammation is a subsequent event during the bimodal stage after injury. The early phase of $I / R$ injury in the spinal cord was found to be largely $\mathrm{TLR}_{4} / \mathrm{MyD}_{88}$-dependent and microglia-dependent, and the following late phase was found to be mainly dependent on $\mathrm{TLR}_{4} / \mathrm{TRIF}$ activation, which was amplified by $\mathrm{MyD}_{88}$ signaling with the involvement of both microglia and astrocytes. These findings explain why several therapeutic treatments have failed in patients with $I / R$ injury. A novel and ideal therapy that would address the different phases of injury and inflammation may be valuable in preventing delayed injury and its clinical manifestations.

\section{Abbreviations}

BSCB: Blood-spinal cord barrier; CD13: Aminopeptidase-N; CD31: Platelet endothelial cell adhesion molecule-1; EB: Evan's Blue; GFAP: Glial Fibrillary acidic protein; Iba-1: Ionized calcium-binding adaptor molecule 1;

IL: Interleukin; I/R: Ischemia-reperfusion; MIP: MyD 88 inhibitory peptide; $\mathrm{MyD}_{88}$ : Myeloid differentiation factor ${ }_{88} ; \mathrm{NF-KB}$ : Nuclear factor kappa-B; TLR4: Toll-like receptor 4 ; TRIF: TIR domain-containing adaptor-inducing IFN- $\beta$.

\section{Competing interests}

The authors declare that they have no competing interests.

\section{Authors' contributions}

$X-Q L$ and BF participated in the animals' care and made all the animal models. X-QL, HW and W-FT participated in tissue preparation, and sectioning and performed most immunohistochemistry; X-QL, BF and W-FT performed Western blotting assay and statistical analysis; $\mathrm{HM}$ involved in the guide of model design and study design; H-WL gave important directions to data analysis and manuscript writing. All authors read and approved the final manuscript.

\section{Acknowledgements}

Funding for this project was provided by Doctoral Fund of Ministry of Education of China, No. 20092104110009 and Natural Science Foundation of China, No. 81271370

Received: 27 November 2013 Accepted: 9 March 2014

Published: 28 March 2014

\section{References}

1. Bell MT, Puskas F, Agoston VA, Cleveland JC Jr, Freeman KA, Gamboni F, Herson PS, Meng X, Smith PD, Weyant MJ, Fullerton DA, Reece TB: Toll-like receptor 4-dependent microglial activation mediates spinal cord ischemia-reperfusion injury. Circulation 2013, 128(1):S152-S156.

2. Zhu P, Li JX, Fujino M, Zhuang J, Li XK: Development and treatment of inflammatory cells and cytokines in spinal cord ischemia-reperfusion injury. Mediators Inflamm 2013, 2013:701970.

3. Smith PD, Puskas F, Meng XZ, Lee JH, Cleveland JC, Weyant MD, Fullerton DA, Reece TB: The evolution of Chemokine release supports a bimodal mechanism of spinal cord ischemia and reperfusion injury. Circulation 2013, 126(suppl 1):S110-S117.

4. Kahn RA, Stone ME, Moskowita DM: Anesthetic consideration for descending thoracic aortic aneurysm repair. Semin Cardiothorac Vasc Anesth 2007, 11:205-223

5. Patel VI, Ergul E, Conrad MF, Cambria M, LaMuraglia GM, Kwolek CJ, Brewster DC, Cambria RP: Continued favorable results with open surgical 
repair of type IV thoracoabdominal aortic aneurysms. J Vasc Surg 2011, 53:1492-1498.

6. Abbott NJ, Rönnbäck L, Hansson E: Astrocyte-endothelial interactions at the blood-brain barrier. Nat Rev Neurosci 2006, 7:41-53.

7. Matsmumoto S, Matsumoto M, Yamashita A, Ohtake K, Ishida K, Morimoto $Y$, Sakabe T: The temporal profile of the reaction of microglia, astrocytes, and macrophages in the delayed onset paraplegia after transient spinal cord ischemia in rabbits. Anesth Analg 2003, 96:1777-1784.

8. Fang B, Li XM, Sun XJ, Bao NR, Ren XY, Lv HW, Ma H: Ischemic preconditioning protects against spinal cord ischemia-reperfusion injury in rabbits by attenuating blood spinal cord barrier disruption. Int J Mol Sci 2013, 14:10343-10354.

9. Fang $B$, Wang $H$, Sun $X J$, Li XQ, Ai CY, Tan WF, White PF, Ma H: Intrathecal transplantation of bone marrow stromal cells attenuates blood-spinal cord barrier disruption induced by spinal cord ischemia-reperfusion injury in rabbits. J Vasc Surg 2013, 58:1043-1052.

10. Tanga FY, Nutile-McMenemy N, Deleo JA: The CNS role of toll-like receptor 4 in innate neuroimmunity and painful neuropathy. Proc Natl Acad SCi US A 2005, 102:5856-5861.

11. Wang J, Hou J, Zhang P, Li D, Zhang C, Liu J: Geniposide reduces inflammatory responses of oxygen-glucose deprived Rat microglial cells via inhibition of the TLR4 signaling pathway. Neurochem Res 2012, 37:2235-2248

12. JaJakus PB, Kalman N, Antus C, Radnai B, Tucsek Z, Gallyas F Jr, Sumegi B, Veres B: TRAF6 is functional in inhibition of TLR4-mediated NF-KB activation by resveratrol. J Nutr Biochem 2013, 24:819-823

13. Hanafy KA: The role of microglia and the TLR4 pathway in neuronal apoptosis and vasospasm after subarachnoid hemorrhage. J Neuroinflammation 2013, 10:83.

14. Puneet P, McGrath MA, Tay HK, Al-Riyami L, Rzepecka J, Moochhala SM, Pervaiz $S$, Harnett MM, Harnett W, Melendez AJ: The helminth product ES-62 protects against septic shock via toll-like receptor 4-dependent autophagosomal degradation of the adaptor MyD88. Nat Immunol 2011, 12:344-351.

15. Oshiumi H, Sasai M, Shida K, Fujita T, Matsumoto M, Seya T: TIR-containing adapter molecule (TICAM)-2, a bridging adapter recruiting to toll-like receptor 4 TICAM-1 that induces interferon- $\beta$. J Biol Chem 2003, 278:49751-49762.

16. Yao AH, Jia LY, Zhang YK, Ma QR, Cheng P, Liu L, Ju G, Kuang F: Early blockade of TLRs MyD88-dependent pathway May reduce secondary spinal cord injury in the rats. Evid Based Complement Alternat Med 2012. 2012:591298.

17. O'Neill LA, Bowie AG: The family of five: TIR-domain-containing adaptors in toll-like receptor signaling. Nat Rev Immunol 2007, 7:353-364.

18. Saito O, Svensson Cl, Buczynski MW, Wegner K, Hua XY, Codeluppi S, Schaloske RH, Deems RA, Dennis EA, Yaksh TL: Spinal glial TLR4-mediated nociception and production of prostaglandin E (2) and TNF. Br J Pharmacol 2010, 160:1754-1764.

19. Gao Y, Fang XB, Sun H, Wang Y, Yao LJ, Li JP, Tong Y, Zhang BH, Liu YL: Toll-like receptor 4-mediated myeloid differentiation factor 88-dependent signaling pathway is activated by cerebral ischemiareperfusion in hippocampal CA1 region in mice. Biol Pharm Bull 2009, 32:1665-1671

20. Xie XH, Zang N, Li SM, Wang LJ, Deng Y, He Y, Yang XQ, Liu EM: Resveratrol Inhibits respiratory syncytial virus-induced IL-6 production, decreases viral replication, and down regulates TRIF expression in airway epithelial cells. Inflammation 2012, 35:1392-1401.

21. Lang-Lazdunski L, Matsushita K, Hirt L, Waeber C, Vonsattel JP, Moskowitz MA, Dietrich WD: Spinal cord ischemia. Development of a model in the mouse. Stroke 2000, 31:208-213.

22. Gong G, Yuan LB, Hu L, Wu W, Yin L, Hou JL, Liu YH, Zhou LS: Glycyrrhizin attenuates rat ischemic spinal cord injury by suppressing inflammatory cytokines and HMGB1. Acta Pharmacol Sin 2012, 33(1):11-18.

23. Safi HJ, Estrera AL, Miller CC, Huynh TT, Porat EE, Azizzadeh A, Meada R, Goodrick JS: Evolution of risk for neurologic deficit after descending and thoracoabdominal aortic repair. Ann Thorac Surg 2005, 80:2173-2179

24. Papakostas JC, Matsagas MI, Toumpoilis IL, Malamous-Mitsi VD, Pappa LS, Gkrepi C, Anagnostopoulos CE, Kappas AM: Evolution of spinal cord injury in a porcine model of prolonged aortic occlusion. J Surg Res 2006 133:159-166.
25. Tsuruta S, Matsumoto M, Fukuda S, Yamashita A, Cui YJ, Wakamatsu H, Sakabe T: The effects of cyclosporin A and insulin on ischemic spinal cord injury in rabbits. Anesth Analg 2006, 102:1722-1727.

26. Zhang YK, Liu JT, Peng ZW, Fan H, Yao AH, Peng C, Liu L, Ju G, Kuang F: Different TLR4 expression and microglia/ macrophage activation induced by hemorrhage in the rat spinal cord after compressive injury. J Neuroinflammation 2013, 10:112.

27. Kim JY, Choi GS, Cho YW, Cho H, Hwang SJ, Ahn SH: Attenuation of spinal cord injury-induced astroglial and microglial activation by repetitive transcranial magnetic stimulation in rats. J Korean Med Sci 2013, 28(2):295-299.

28. Costello DA, Lynch MA: Toll-like Receptor 3 activation modulates Hippocampal network excitability, via glial production of interferon- $\beta$. Hippocampus 2013, 23(8):696-707.

29. Zughaier SM, Zimmer SM, Datta A, Carlson RW, Stephens DS: Differential induction of the toll-like receptor 4-MyD88-dependent and -independent signaling pathways by endotoxins. Infect Immun 2005, 73:2940-2950.

30. Wang S, Schmaderer C, Kiss E, Schmidt C, Bonrouhi M, Porubsky S, Gretz N, Schaefer L, Kirschning CJ, Popovic ZV, Gröne HJ: Recipient Toll-like receptors contribute to chronic graft dysfunction by both MyD88and TRIF-dependent signaling. Dis Model Mech 2010, 3:92-103.

31. Fitzgerald KA, Rowe DC, Barnes BJ, Caffrey DR, Visintin A, Latz E, Monks B, Pitha PM, Golenbock DT: LPS-TLR4 signaling to IRF-3/7 and NF-kappaB involves the toll adapters TRAM and TRIF. J Exp Med 2003, 198:1043-1055.

32. Stroo I, Stokman G, Teske GJ, Raven A, Butter LM, Florquin S, Leemans JC Chemokine expression in renal ischemia/reperfusion injury is most profound during the reparative phase. Int Immunol 2010, 22:433-442.

33. Dinarello CA: A clinical perspective of IL-1 $\beta$ as the gatekeeper of inflammation. Eur I Immunol 2011, 41:1203-1217.

34. Akuzawa S, Kazui T, Shi E, Yamashita K, Bashar MA, Terada H: Interleukin-1 receptor antagonist attenuates the severity of spinal cord ischemic injury in rabbits. J Vasc Surg 2008, 48:694-700.

doi:10.1186/1742-2094-11-62

Cite this article as: Li et al:: Role of the TLR4 pathway in blood-spinal cord barrier dysfunction during the bimodal stage after ischemia/ reperfusion injury in rats. Journal of Neuroinflammation 2014 11:62.

\section{Submit your next manuscript to BioMed Central and take full advantage of:}

- Convenient online submission

- Thorough peer review

- No space constraints or color figure charges

- Immediate publication on acceptance

- Inclusion in PubMed, CAS, Scopus and Google Scholar

- Research which is freely available for redistribution 